\title{
Is chemotherapy necessary for patients with molar pregnancy and human chorionic gonadotropin serum levels raised but falling at 6 months after uterine evacuation?
}

\author{
Antonio Braga ${ }^{\mathrm{a}, \mathrm{b}, \mathrm{c}, *}$, Berenice Torres ${ }^{\mathrm{a}, \mathrm{c}}$, Marcelo Burlá ${ }^{\mathrm{a}, \mathrm{b}}$, Izildinha Maestá ${ }^{\mathrm{d}}$, Sue Yazaki Sun ${ }^{\mathrm{e}}$, Lawrence Lin ${ }^{\mathrm{f}}$, \\ José Mauro Madi ${ }^{g}$, Elza Uberti ${ }^{\mathrm{h}}$, Maurício Viggiano ${ }^{\mathrm{i}},{\text { Kevin M. } \text { Elias }^{\mathrm{j}} \text {, Ross S Berkowitz }}^{\mathrm{j}}$

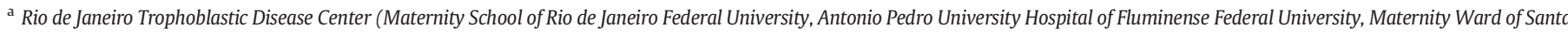 \\ Casa da Misericórdia do Rio de Janeiro), Rio de Janeiro, Rio de Janeiro, Brazil \\ b Postgraduate Program in Medical Sciences, Fluminense Federal University, Niterói, Rio de Janeiro, Brazil \\ ${ }^{c}$ Postgraduate Program in Perinatal Health, Faculty of Medicine, Maternity School of Rio de Janeiro Federal University, Brazil \\ d Trophoblastic Diseases Center of the Clinical Hospital of Botucatu Medical School, Department of Gynecology and Obstetrics, São Paulo State University, Botucatu, São Paulo, Brazil \\ e São Paulo Hospital Trophoblastic Disease Center, Paulista School of Medicine, São Paulo Federal University, São Paulo, São Paulo, Brazil \\ f São Paulo Clinics Hospital Trophoblastic Disease Center, University of São Paulo, São Paulo, São Paulo, Brazil

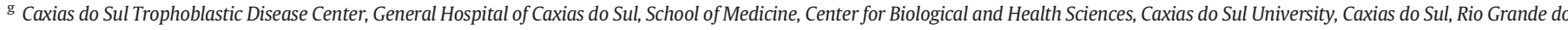 \\ Sul, Brazil \\ h Porto Alegre Trophoblastic Disease Center, Mario Totta Maternity Ward, Irmandade da Santa Casa de Misericórdia Hospital, Porto Alegre, Rio Grande do Sul, Brazil \\ ${ }^{i}$ Goiás Trophoblastic Disease Center, Clinical Hospital of Goiás, Goiás Federal University, Goiânia, Goiás, Brazil

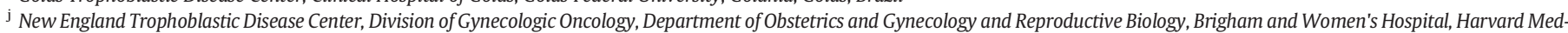 \\ ical School, Boston, MA, USA
}

\section{H I G H L I G H T S}

- Only $0.8 \%$ of women have raised but falling hCG 6 months post molar evacuation.

- More than $80 \%$ of such cases will enter remission without further therapy.

- GTN treated after observation has comparable outcomes to immediate chemotherapy.

\section{A R T I C L E I N F O}

\section{Article history:}

Received 3 July 2016

Received in revised form 6 September 2016

Accepted 10 September 2016

\section{Keywords:}

Molar pregnancy

Human chorionic gonadotropin

Chemotherapy

\section{A B S T R A C T}

Objective. To compare the outcomes of Brazilian patients with molar pregnancy who continue human chorionic gonadotropin (hCG) surveillance with those treated with chemotherapy when hCG was still positive, but falling at 6 months after uterine evacuation.

Methods. Retrospective chart review of 12,526 patients with hydatidiform mole treated at one of nine Brazilian reference centers from January 1990 to May 2016.

Results. At 6 months from uterine evacuation, $96(0.8 \%)$ patients had hCG levels raised but falling. In 15/96 (15.6\%) patients, chemotherapy was initiated immediately per FIGO 2000 criteria, while 81/96 (84.4\%) patients were managed expectantly. Among the latter, 65/81 (80.2\%) achieved spontaneous remission and 16 (19.8\%) developed postmolar gestational trophoblastic neoplasia (GTN). Patients who received chemotherapy following expectant management required more time for remission ( 11 versus 8 months; $p=0.001$ ), had a greater interval between uterine evacuation and initiating chemotherapy ( 8 versus 6 months; $p<0.001$ ), and presented with a median WHO/FIGO risk score higher than women treated according to FIGO 2000 criteria (4 versus 2, $p=$ $0.04)$, but there were no significant differences in the need for multiagent treatment regimens ( $1 / 15$ versus $3 /$ 16 patients, $p=0.60$ ). None of the women relapsed, and no deaths occurred in either group.

Conclusion. In order to avoid unnecessary exposure of women to chemotherapy, we no longer follow the FIGO 2000 recommendation to treat all patients with molar pregnancy and hCG raised but falling at 6 months after evacuation. Instead, we pursue close hormonal and radiological surveillance as the best strategy for these patients. (c) 2016 Elsevier Inc. All rights reserved.

\footnotetext{
* Corresponding author at: Gestational Trophoblastic Disease Center of Rio de Janeiro. Maternidade Escola, Universidade Federal do Rio de Janeiro, Rua Laranjeiras, 180, Laranjeiras, Rio de Janeiro 22240-003, Brazil.

E-mail address: bragamed@yahoo.com.br (A. Braga).
} 


\section{Introduction}

Gestational trophoblastic disease (GTD) is an abnormality of pregnancy that includes benign clinical forms (complete hydatidiform moles - CHM and partial hydatidiform moles - PHM) with variable potential to develop gestational trophoblastic neoplasia (GTN), in its various spectra (invasive mole, choriocarcinoma, placental site trophoblastic tumor, and epithelioid trophoblastic tumor) [1-3]. Although GTD develops in 1:1000 pregnancies in North America and 1:2000 pregnancies in Europe, it is estimated to occur five to ten times more frequently in Brazil [4,5]. Serial monitoring of human chorionic gonadotropin (hCG) levels after molar uterine evacuation is essential to detect malignant progression and initiate chemotherapy [6]. In Brazil, despite many difficulties, this postmolar follow-up is done in 38 GTD Reference Centers, spread throughout this country of continental dimensions $[7,8]$.

The Brazilian Reference Centers of GTD have adopted the criteria established by the International Federation of Gynecology and Obstetrics (FIGO) for the diagnosis of postmolar GTN: rising (>10\%) hCG levels for three consecutive weeks or plateaued for four weeks, if there is a histologic diagnosis of choriocarcinoma, or when the hCG level remains elevated for 6 months or more from the molar uterine evacuation [9]. The most common reason for the diagnosis of postmolar GTN and the start of chemotherapy is a plateau or rise in hCG values [10]. However, some investigators have indicated that it is safe to maintain hormonal surveillance among patients with molar pregnancy whose hCG levels are raised but falling beyond the 6 months after uterine evacuation and that spontaneous remission will occur in most cases without chemotherapy [10-15].

While some organizations, such as the European Organisation for Treatment of Trophoblastic Diseases, argue that the presence of hCG raised but falling in the 6th month after molar evacuation is not an indication to begin chemotherapy, there appears to be a controversy $[2,16]$. FIGO initially advised initiating chemotherapy when hCG was elevated but falling at 6 months after molar evacuation in 2000, retracted this opinion in 2012, but then resumed the recommendation in the FIGO Cancer Report in 2015 [9,17,18].

We must be cautious before dismissing this FIGO recommendation since postponing the chemotherapy in these patients with molar pregnancy and hCG levels present over 6 months after uterine evacuation could worsen the prognosis of the GTN, due to the potential development of tumor mutations and consequent chemoresistance (an important cause of mortality among Brazilian women with GTN) [4]. Furthermore, delay could increase the FIGO risk score for these patients as the "interval between antecedent pregnancy and start of chemotherapy" is included in the current FIGO/WHO Prognostic Scoring System, which would mean higher risk scores and potentially more treatment with multiagent regimens for patients due to delayed initiation of treatment [19-22].

The present study was undertaken to compare the outcomes of Brazilian patients with molar pregnancy that continued hCG surveillance with those treated with chemotherapy when hCG was still positive, but falling at 6 months after uterine evacuation. We also wanted to identify risk factors that were associated with the development of GTN in patients who continued hCG monitoring. The study was initiated to advance our understanding if it was prudent to continue hCG monitoring versus initiating chemotherapy in patients with elevated but falling hCG levels 6 months after molar evacuation.

\section{Material and methods}

\subsection{Study design}

This is a retrospective cohort study of patients with molar pregnancy followed at one of nine Brazilian GTD Reference Centers: in Rio de Janeiro (Maternity School of Rio de Janeiro Federal University, Antonio
Pedro University Hospital of Fluminense Federal University, Maternity Ward of Santa Casa da Misericórdia do Rio de Janeiro - data entered by $A B$ and audited by MB), in Goiânia (Clinical Hospital of Goiás Federal University - data entered by MV and audited by MB), in Porto Alegre (Mario Totta Maternity Ward at Irmandade da Santa Casa de Misericórdia Hospital - data entered by EU and audited by MB), in Caxias do Sul (General Hospital of Caxias do Sul - data entered by JMM and audited by MB), in São Paulo (São Paulo Hospital of São Paulo Federal University and São Paulo Clinics Hospital of University of São Paulo data entered by SYS and LL and audited by MB) and in Botucatu (Clinical Hospital of São Paulo State University - data entered by IM and audited by MB) from January 1990 to May 2016.

\subsection{Study participants}

Molar pregnancy was suspected when women presented with symptoms of pregnancy or vaginal bleeding combined with an elevated hCG level. The diagnosis of molar pregnancy in all cases was strengthened by sonographic evaluation of the uterus. All patients had uterine evacuation performed using suction curettage with histopathological confirmation of CHM or PHM.

This study includes all patients treated at one of the above centers during the study period, who adhered to the postmolar follow-up and presented with hCG levels raised but falling at 6 months after uterine evacuation, and whose medical records were complete and available for review. The following patients were excluded: those with nonmolar pregnancy; who had hysterectomy to treat molar pregnancy (12 patients); those who became pregnant during the postmolar follow-up (148 patients) or were lost to postmolar follow-up (178 patients); with histological diagnosis of choriocarcinoma (98 patients), placental site trophoblastic tumor (26 patients) or epithelioid trophoblastic tumor ( 9 patients); and women later found to have false-positive or ("phantom") hCG (36 patients).

\subsection{Postmolar follow-up}

Postmolar follow-up, in addition to contraception as suggested and provided to patients, consisted of clinical examinations and laboratory tests, including the measurement of hCG levels until gonadotropin remission. Remission was defined as three consecutive weekly results of hCG values below $5 \mathrm{IU} / \mathrm{L}$. After that, medical visits and measurement of hCG levels continued monthly for six months in the case of spontaneous remission. After chemotherapy, all the patients underwent follow-up for at least a year with monthly hCG surveillance after the first normal hCG value was obtained. When patients did not attend the scheduled visits, a social worker and hospital psychologist actively tried to contact them by phone and telegram to identify what was hindering compliance and to motivate them to return to follow-up.

The patients with molar pregnancy with hCG levels raised but falling at 6 months after uterine evacuation and not immediately treated with chemotherapy were strictly followed with weekly hCG measurement and monthly chest radiograph and Doppler ultrasound scan of the pelvis in order to detect invasive mole or early metastasis. Until the year 2000 , all patients were systematically treated with chemotherapy in these cases. After that, the adoption of expectant management or immediate treatment of these patients was made after medical discussion, informed consent and individualization of the case. No formal randomization was used.

Measurement in all nine Reference Centers employed the Siemens Diagnostic Products Corporation (DPC) Immulite ${ }^{\circledR}$ assay. The reference value for normal results was an hCG value below $5 \mathrm{IU} / \mathrm{L}$. 
2.4. Diagnosis, staging, risk factors and treatment of gestational trophoblastic neoplasia

Malignant progression was diagnosed using the criterion established by FIGO 2000 [9]. Lung metastases were detected using a chest X-ray, although computed tomography scan was also sometimes used as an aid in follow-up and treatment [9]. Nuclear magnetic resonance imaging of the brain and abdomen was used for patients where pulmonary lesions were detected. GTN was staged according to the FIGO 2000 criteria: I disease confined to the uterus; II - disease extends to the outside of the uterus, but is limited to the genital structures; III - disease extends to the lungs, with or without genital tract involvement; and IV - all other metastatic sites. Prognostic scoring for resistance to chemotherapy followed the FIGO/WHO Prognostic Scoring System [9].

Methotrexate and folinic acid rescue (MTX/FA) $1 \mathrm{mg} / \mathrm{kg}$ intramuscular and $0.1 \mathrm{mg} / \mathrm{kg}$ per oral, respectively, was used as a first line treatment in all cases of low-risk GTN. In cases of chemoresistance, second line chemotherapy was performed with actinomycin-D (Act-D) $1.25 \mathrm{mg}$ intravenous pulse every 15 days. The third line chemotherapy treatment used was the EMA/CO regimen (etoposide, MTX/FA, Act-D, cyclophosphamide, and vincristine).

\subsection{Outcomes}

The primary outcome was the occurrence of GTN among the study population. Secondary outcomes included the occurrence of metastases, the presence of high risk FIGO score, the need of multiagent chemotherapy, time to eventual remission, and occurrence of death.

\subsection{Variables}

Collected patient variables included the patient age (years), hCG level preevacuation (IU/L), the gestational age at diagnosis based on date of last menstruation (weeks), the histology of molar pregnancy (CHM or PHM), hCG level at 6 months after molar evacuation (IU/L), and the time to hCG level normalization (months). Among cases of GTN, selected variables included the time interval between the uterine evacuation and the beginning of chemotherapy (months), the presence of metastasis, the presence of high risk FIGO score, the treatment with multiagent chemotherapy, the number of chemotherapy cycles, and the occurrence of death.

\subsection{Statistical analysis}

Normality among continuous variables was tested using the D'Agostino-Pearson omnibus test. Normally distributed data were analyzed using a Student's $t$-test, while non-normally distributed data were analyzed using the Mann-Whitney $U$ test. The Chi-square and Fisher's exact tests were used to compare proportions. The alpha level for significance for all tests was set at 0.05. InStat GraphPad software was used to perform statistical analyses (GraphPad InStat version 6.0, GraphPad Software, San Diego, CA).

\subsection{IRB approval}

This study was approved by the local Institutional Review Board of the Maternity School of the Federal University of Rio de Janeiro, associated with the Brazilian Research Ethics Committee, under protocol number 572,887 (CAAE 23129813.0.1001.5275).

\section{Results}

Fig. 1 represents a flow diagram describing the study population. From January 1990 to May 2016, 12,526 patients were treated at the participating Reference Centers for molar pregnancy. Among these, 8899 (71\%) achieved hCG remission spontaneously within 6 months of uterine evacuation, 3531 (28.2\%) developed postmolar GTN and 96 $(0.8 \%)$ patients had hCG levels raised but falling 6 months after uterine evacuation and were included in our study.

Considering only patients with hCG levels elevated at 6 months after uterine evacuation, we observed that 15 (15.6\%) patients were treated with chemotherapy based on the FIGO 2000 criteria, and 81 (84.4\%) patients were followed with expectant management. Among the $81 \mathrm{pa}-$ tients who were followed, 65 (80.2\%) patients achieved spontaneous remission, and 16 (19.8\%) developed late postmolar GTN (due to plateau or rising hCG levels). None of the women relapsed, and no deaths have occurred.

Table 1 summarizes the characteristics of Brazilian women with raised but falling human chorionic gonadotropin serum levels at 6 months after uterine evacuation not treated with chemotherapy, despite the FIGO 2000 criteria. Median age (29 versus 25 years old; $p=$ 0.11 ) and median gestational age at diagnosis (10 versus 12 weeks; $p=0.13$ ) among women in this group achieving spontaneous remission and progressing to GTN were similar. However, when compared to women who later went on to develop GTN, those women who achieved spontaneous remission had median lower pre-evacuation hCG levels (100,000 versus 225,000 IU/L; $p=0.02$ ) and median lower hCG levels at 6 months after uterine evacuation (68 versus $546 \mathrm{IU} / \mathrm{L}$; $p=0.02$, also shown in Fig. 2). There was also a trend towards a lower prevalence of the histologic diagnosis of CHM (67.7 versus 93.8\%; $p=0.06$ ), although this did not reach statistical significance. No deaths were reported.

The characteristics of all women from the study population treated with chemotherapy, whether at 6 months following uterine evacuation or later due to a plateau or rising hCG, are summarized in Table 2 and Fig. 3. Patients that received chemotherapy due to FIGO 2000 criteria were older than those treated due to late progression GTN ( 32 versus 25 years old; $p=0.01$ ). There were no differences between these groups, in terms of the median preevacuation hCG level (219,651 versus $225,000 \mathrm{IU} / \mathrm{L} ; p=0.51$ ), median gestational age at diagnosis (12 versus 12 weeks of gestation; $p=0.96$ ), the histologic diagnosis of CHM (86.6 versus $93.8 \%$; $p=0.60$ ), median hCG level at 6 months after evacuation ( 190 versus $546 \mathrm{IU} / \mathrm{L} ; p=0.26$ ), occurrence of metastasis ( 13.3 versus $12.5 \%$; $p=1.0$ ), or duration of follow up (24 versus 36 months; $p=$ 0.56). Patients with a diagnosis of late GTN, when compared with those treated due to FIGO 2000 criteria, did require more time for hCG level normalization ( 11 versus 8 months; $p=0.001$ ) and had a greater interval between uterine evacuation and beginning of chemotherapy ( 8 versus 6 months; $p<0.001$ ). Consequently, the median WHO/FIGO risk score was higher among women receiving chemotherapy for late progression to GTN ( 4 versus $2, p=0.04$ ), but there were no significant differences either in the total number of chemotherapy cycles (single agent: 4 (4-8) versus 3 (3-7); $p=0.48$ and multiagent regimen: 5 (4-8) versus $4(4) ; p=0.51$ ) or the need for multiagent treatment regimens ( $3 / 16$ patients versus $1 / 15, p=0.60)$ to achieve remission. There were no reported cases of relapsed GTN or death.

\section{Discussion}

We have shown that it appears to be safe to follow patients with molar pregnancy and hCG serum levels raised but falling at 6 months after uterine evacuation, with $80.2 \%$ of these patients achieving hormonal remission in a median of 8 months. Our observation is similar to others, but is in contrast to FIGO criteria that recommend the beginning of chemotherapy treatment for these patients [9-15].

We would like to highlight that our data come from a region where no similar work has been previously reported. Agarwal et al. identified retrospectively 75/13,910 (0.5\%) patients with molar pregnancy treated at Charing Cross Hospital with persistent but falling hCG at 6 months after uterine evacuation [10]. Among 66 patients with hormonal surveillance, 65 (98\%) went to spontaneous remission. Similar to our results, they found higher median hCG levels at 6 months after 


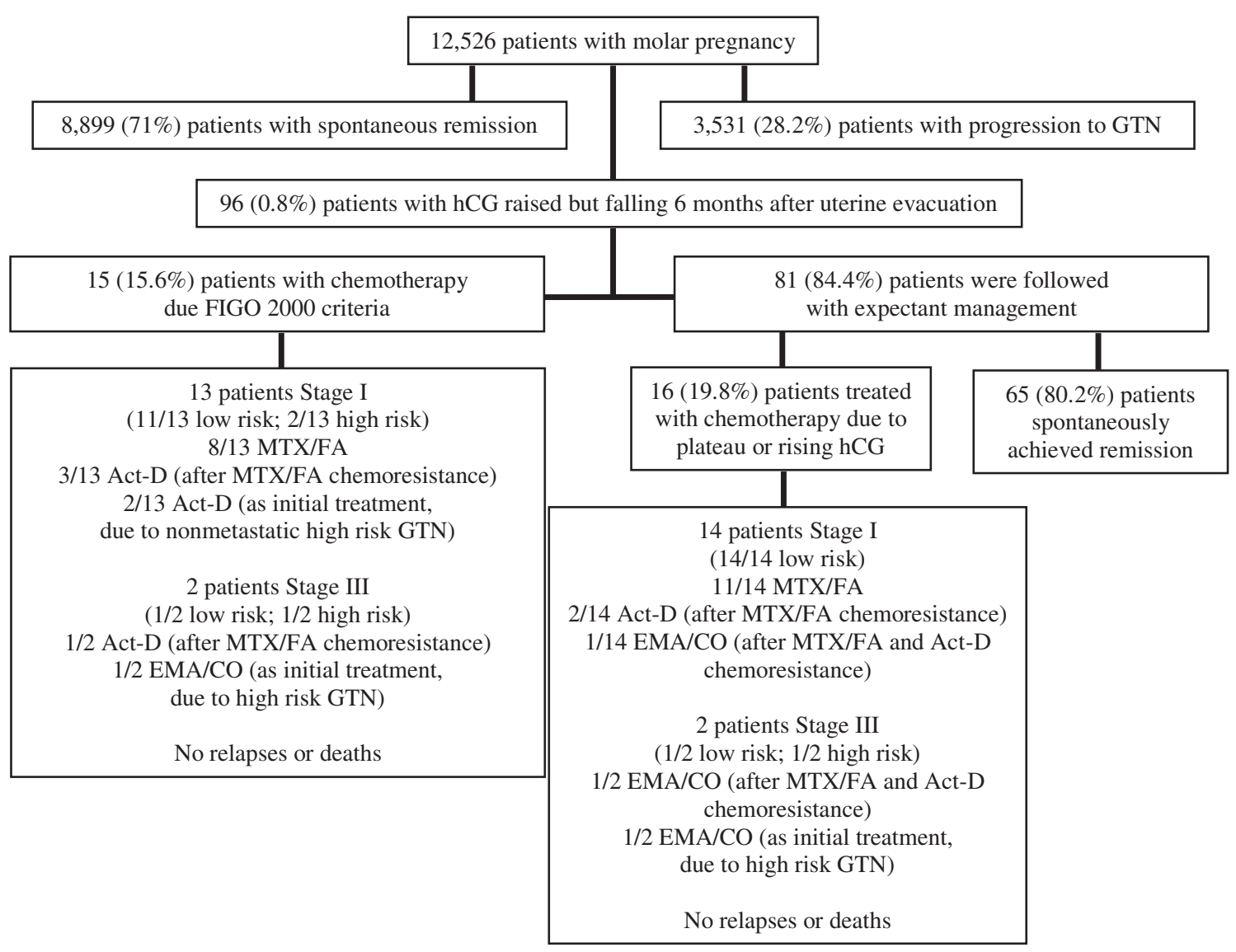

Fig. 1. Flow diagram summarizing the derivation of the study population.

evacuation in those women who developed late GTN (13 IU/L, range 5887 versus $157 \mathrm{IU} / \mathrm{L}$, range $6-6438 ; p=0.004)$. However, all patients attained remission, and they did not report any relapse of GTN. Recently, new data from the United Kingdom Trophoblastic supra-regional disease treatment centers at Weston Park Hospital (Sheffield) and Charing Cross Hospital (London) reported 35/16,779 (0.2\%) women with molar pregnancy and raised but falling serum hCG levels followed with continued surveillance 6 months after evacuation [15]. The authors observed hormonal remission in 30 of the patients (86\%) spontaneously, similar to our results $(80.2 \%)$; one woman was lost to follow up prior to hCG normalization (3\%) and the remaining four women (11\%) were treated with chemotherapy due to late GTN. No woman developed relapsed disease and overall survival was $100 \%$.

Our investigation reinforces previous findings in the literature showing that the occurrence of hCG levels raised but falling at 6 months after uterine evacuation is extremely low, affecting $<1 \%$ of patients with molar pregnancy. In such cases, it appears to be safe to maintain hormonal and radiological surveillance, since approximately $80 \%$ of patients will normalize the levels of hCG spontaneously [10-15]. However, the current study also adds some new findings. Among patients not treated with chemotherapy, despite FIGO 2000 criteria, with raised but falling hCG serum levels at 6 months after uterine evacuation,

Table 1

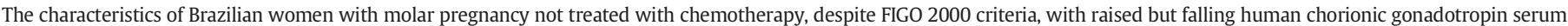
levels at 6 months after uterine evacuation.

\begin{tabular}{|c|c|c|c|}
\hline \multirow[b]{2}{*}{ Variable } & \multicolumn{2}{|c|}{ Outcome after observation at 6 months post-evacuation } & \multirow[b]{2}{*}{$p$-Value } \\
\hline & Spontaneous remission $\mathrm{N}=65(80.2 \%)$ & $\begin{array}{l}\text { Late progression to GTN } \\
\mathrm{N}=16 \text { patients }(19.8 \%)\end{array}$ & \\
\hline Age, years; median (range) & $29(14-47)$ & $25(14-49)$ & 0.11 \\
\hline hCG preevacuation level, IU/L; median (range) & $100,000(800-900,000)$ & $225,000(1800-567,340)$ & 0.02 \\
\hline Gestational age at diagnosis, weeks, median (range) & $10(4-28)$ & $12(6-23)$ & 0.13 \\
\hline \multicolumn{4}{|l|}{ Histology of molar pregnancy } \\
\hline Complete hydatidiform mole, $\mathrm{n}(\%)$ & $44(67.7 \%)$ & $15(93.8 \%)$ & \\
\hline Partial hydatidiform mole, $\mathrm{n}(\%)$ & $21(32.3 \%)$ & $1(6.2 \%)$ & 0.06 \\
\hline hCG level 6 months after evacuation, IU/L; median (range) & $68(6-1750)$ & $546(7-3258)$ & 0.02 \\
\hline Time to hCG level normalization, months; median (range) & $8(6-36)$ & $11(7-26)$ & 0.002 \\
\hline Duration of follow up, months; median (range) & $14(9-46)$ & $36(14-67)$ & $<0.001$ \\
\hline Occurrence of relapse or death, $\mathrm{n}(\%)$ & $0(0)$ & $0(0)$ & - \\
\hline
\end{tabular}

GTN - gestational trophoblastic neoplasia.

hCG - human chorionic gonadotropin. 


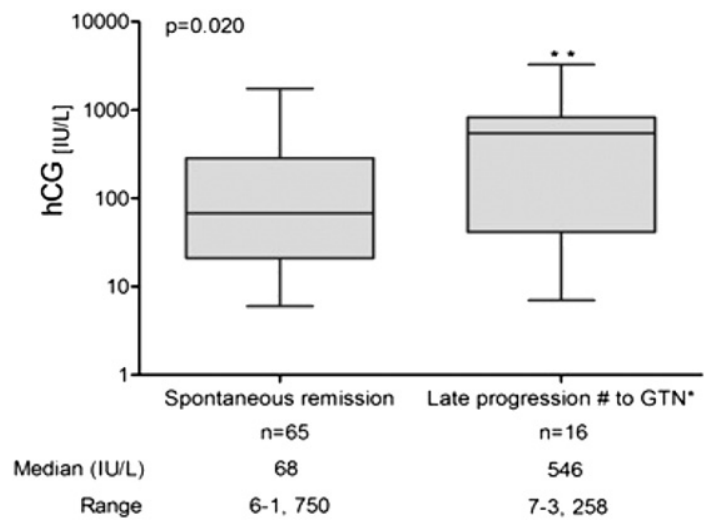

Fig. 2. Human chorionic gonadotropin levels raised but falling among Brazilian women with molar pregnancy at 6 months after uterine evacuation, not treated with chemotherapy, despite FIGO 2000 criteria.

the histology of CHM and higher hCG level preevacuation at 6 months from evacuation were associated with an increased risk of developing late GTN. Patients who were followed with hormonal surveillance and developed late GTN were more likely to have a higher WHO/FIGO prognostic risk score, mostly due to the increased time between evacuation and chemotherapy. Interestingly, however, this delay did not worsen the GTN extent of disease or the response to treatment and did not increase the need for a multiagent regimen.

Our study does have some important limitations. Data were collected from different hospital databases and may not reflect the Brazilian general population. As referral centers, these data may overestimate the true prevalence of GTN in the general population. A commercial kit designed to diagnose pregnancy was used to measure hCG levels, and not to discriminate all its fractions. Despite investigating a large series of patients with molar pregnancy, the low incidence of cases of patients with raised but falling hCG serum levels at 6 months after uterine evacuation is a bias that should be considered.

In conclusion, in settings where patients with molar pregnancy can be closely followed with hormonal and radiological surveillance, we believe it is appropriate to adopt the strategy to "watch and wait", because the hCG levels in the vast majority of women will decrease to normal [11]. These cases should be evaluated in GTD reference centers and must be discussed individually within specialized teams to decide with the patient and her family on the best therapeutic strategy. Continued surveillance avoids exposing women unnecessarily to the potential toxicities of chemotherapy $[10,21,22]$. In our series, we should emphasize that expectant management spared $80 \%$ of patient's unnecessary chemotherapy and its side effects, although methotrexate and actinomycin-D are generally well tolerated. Moreover, patients, who were monitored with hCG surveillance and developed late GTN, had no increased risk for metastatic disease and no increased need for multiagent chemotherapy. Therefore, when patients with molar pregnancy have elevated but falling hCG levels at 6 months after evacuation, we believe it is most reasonable to continue hCG follow up and not initiate immediate chemotherapy treatment.

\section{Conflict of interests}

The authors declare no conflict of interests.

\section{Funding}

This research was supported by the Carlos Chagas Filho Foundation for Research Support in the State of Rio de Janeiro/Brazil (FAPERJ) - an agency under the Brazilian Ministry of Science and Technology (AB); and the Donald P. Goldstein MD Trophoblastic Tumor Registry Endowment (KME, RSB) and the Dyett Family Trophoblastic Disease Research and Registry Endowment (KME, RSB). The funding agencies had no direct role in the generation of the data or manuscript.

Table 2

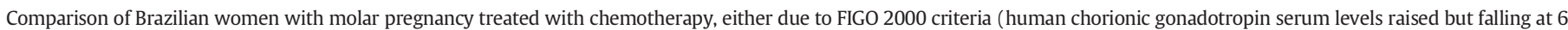
months after uterine evacuation) or later due to progression to GTN.

\begin{tabular}{|c|c|c|c|}
\hline \multirow[b]{2}{*}{ Variable } & \multicolumn{2}{|l|}{ Indication for chemotherapy } & \multirow[b]{2}{*}{$p$-Value } \\
\hline & Due to FIGO 2000 criteria $N=15$ patients & $\begin{array}{l}\text { Due to late GTN } \\
\mathrm{N}=16 \text { patients }\end{array}$ & \\
\hline Age, years; median (range) & $32(17-49)$ & $25(14-49)$ & 0.01 \\
\hline hCG preevacuation level, IU/L; median (range) & $219,651(73,444-745,000)$ & $225,000(1800-567,340)$ & 0.51 \\
\hline Gestational age at diagnosis, weeks, median (range) & $12(9-16)$ & $12(6-23)$ & 0.96 \\
\hline \multicolumn{4}{|l|}{ Histology of molar pregnancy } \\
\hline Complete hydatidiform mole & $13 / 15(86.6 \%)$ & $15 / 16(93.8 \%)$ & 0.60 \\
\hline Partial hydatidiform mole & $2 / 15(13.4 \%)$ & $1 / 16(6.2 \%)$ & \\
\hline hCG level 6 months after evacuation, IU/L; median (range) & $190(37-1130)$ & $546(7-3258)$ & 0.26 \\
\hline Time to hCG level normalization, months; median (range) & $8(7-10)$ & $11(7-26)$ & 0.001 \\
\hline Time between evacuation and chemotherapy, months; median (range) & $6(6-8)$ & $8(6-10)$ & $<0.001$ \\
\hline hCG level pre-treatment, IU/L; median (range) & $240(42-1890)$ & $610(21-3890)$ & 0.30 \\
\hline \multicolumn{4}{|l|}{ Occurrence of metastasis } \\
\hline No, $\mathrm{n}(\%)$ & $13(86.7 \%)$ & $14(87.5 \%)$ & 1.0 \\
\hline Yes, n (\%) & $2(13.3 \%)$ & $2(12.5 \%)$ & \\
\hline WHO/FIGO Prognostic Risk Score & $2(1-9)$ & $4(2-13)$ & 0.04 \\
\hline Low risk, n (\%) & $12(80 \%)$ & $15(93.8 \%)$ & 0.33 \\
\hline High risk, n (\%) & $3(20 \%)$ & $1(6.2 \%)$ & \\
\hline \multicolumn{4}{|l|}{ Treatment } \\
\hline Single agent, $\mathrm{n}(\%)$ & $14(93.3 \%)$ & $13(81.3 \%)$ & 0.60 \\
\hline Multiagent regimen, $\mathrm{n}(\%)$ & $1(6.7 \%)$ & $3(18.7 \%)$ & \\
\hline \multicolumn{4}{|l|}{ Number of chemotherapy cycles necessary to GTN remission } \\
\hline Single agent, median (range) & $3(3-7)$ & $4(4-8)$ & 0.48 \\
\hline Multiagent regimen, median (range) & $4(4)$ & $5(4-8)$ & 0.51 \\
\hline Duration of follow up, months; median (range) & $24(12-84)$ & $36(14-67)$ & 0.56 \\
\hline Occurrence of relapse or death, $n$ & $0(0)$ & $0(0)$ & - \\
\hline
\end{tabular}

GTN - gestational trophoblastic neoplasia.

hCG - human chorionic gonadotropin.

WHO/FIGO - World Health Organization/International Federation of Gynecology and Obstetrics. 
Treatment according to FIGO 2000 Criteria

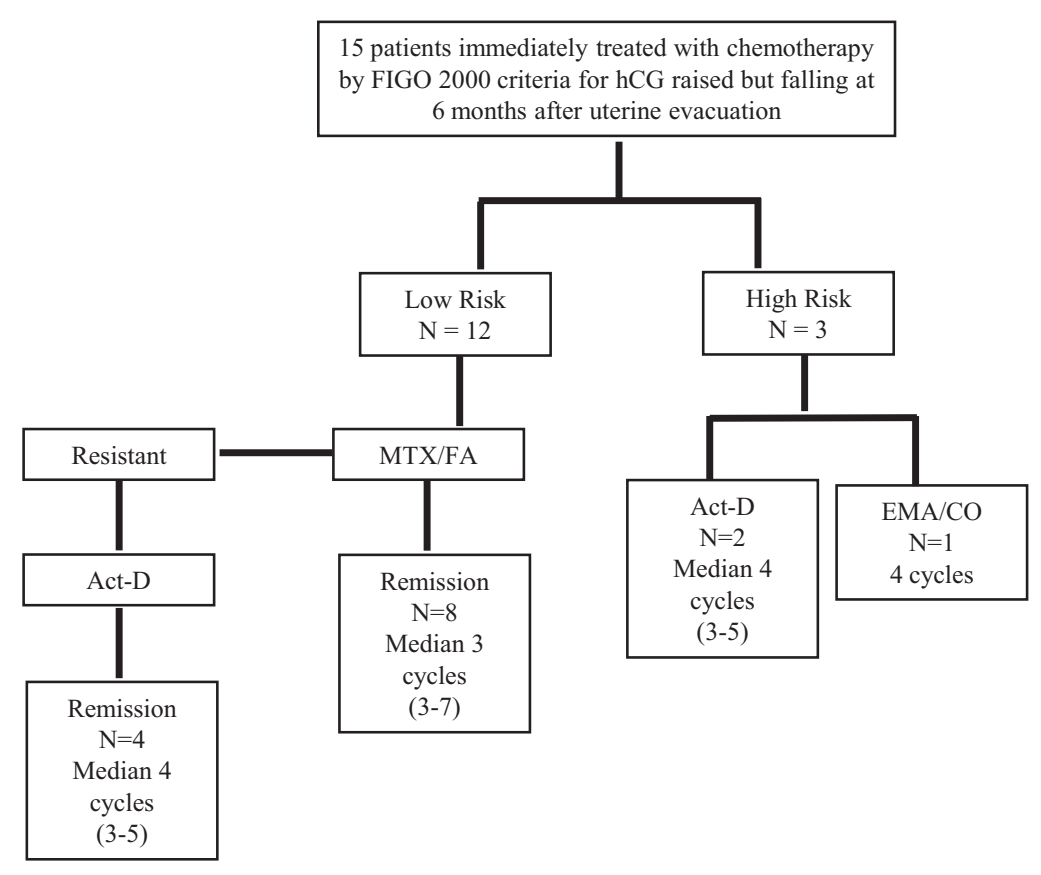

Treatment after Expectant Management

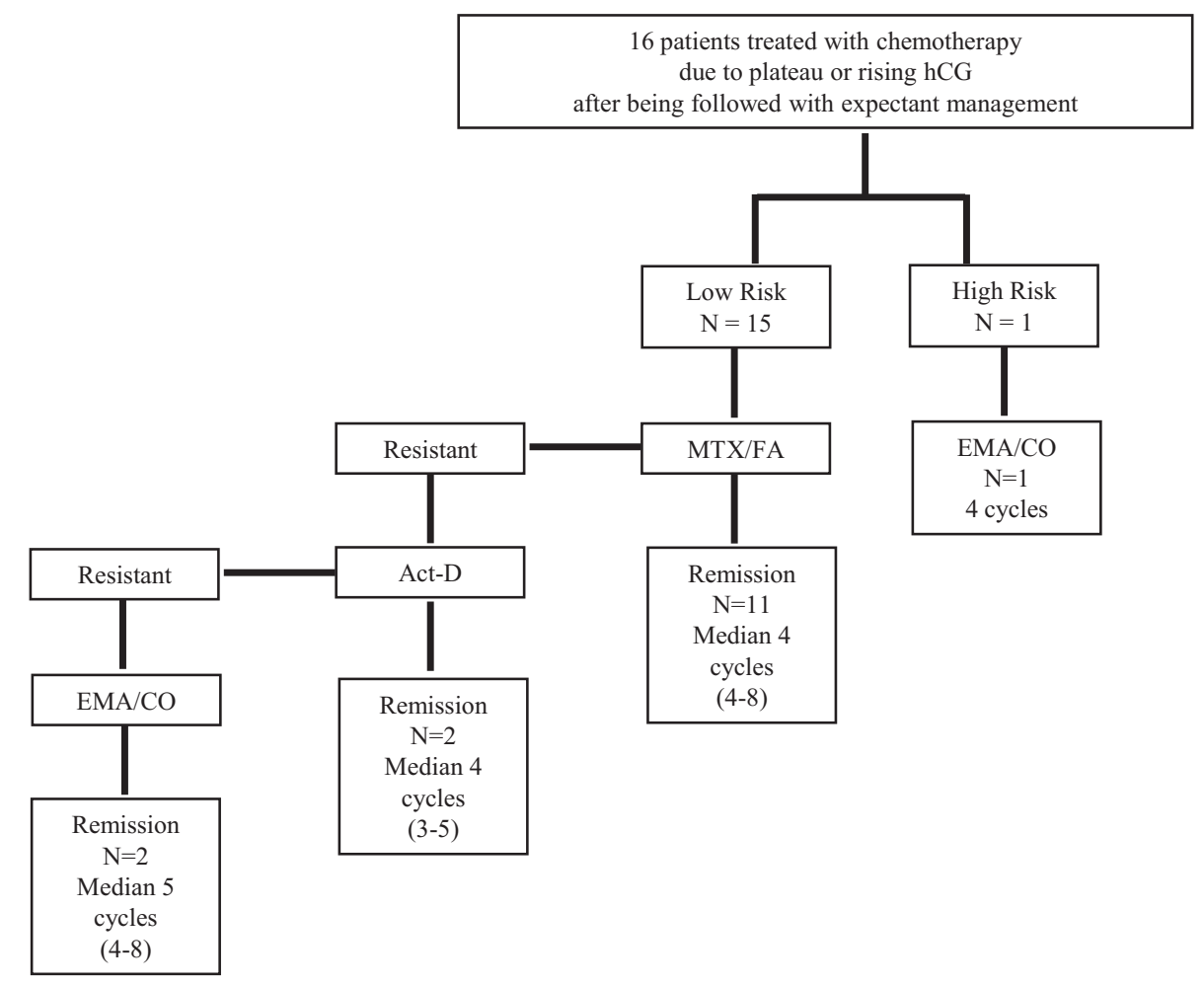

Fig. 3. Flow diagram summarizing the chemotherapy treatment of the study population. Median number of total chemotherapy cycles indicated for each treatment pathway with range in parentheses. 


\section{References}

[1] J. Lurain, Gestational trophoblastic disease I: epidemiology, pathology, clinical presentation and diagnosis of gestational trophoblastic disease, and management of hydatidiform mole, Am. J. Obstet. Gynecol. 203 (6) (2010) 531-539.

[2] M.J. Seckl, N.J. Sebire, R.A. Fisher, F. Golfier, L. Massuger, C. Sessa, ESMO guidelines working group. Gestational trophoblastic disease: ESMO clinical practice guidelines for diagnosis, treatment and follow-up, Ann. Oncol. 24 (Suppl. 6) (2013) vi39-vi50.

[3] A. Biscaro, A. Braga, R.S. Berkowitz, Diagnosis, classification and treatment of gestational trophoblastic neoplasia, Rev. Bras. Ginecol. Obstet. 128 (1) (2013) 3-5.

[4] A. Braga, E. Uberti, M.C. Fajardo, M. Viggiano, S. Sun, B. Grillo, et al., Epidemiological report on the treatment of patients with gestational trophoblastic disease in $10 \mathrm{Bra}-$ zilian referral centers. Results after 12 years since International FIGO 2000 consensus, J Reprod Med 59 (5-6) (2014) 241-247.

[5] Y.K. Eysbouts, J. Bulten, P.B. Ottevanger, C.M. Thomas, M.J. Ten Kate-Booij, A.E. van Herwaarden, et al., Trends in incidence for gestational trophoblastic disease over the last 20 years in a population-based study, Gynecol. Oncol. 140 (1) (2016) 70-75

[6] R.S. Berkowitz, D.P. Goldstein, Current advances in the management of gestational trophoblastic disease, Gynecol. Oncol. 128 (1) (2013) 3-5.

[7] I. Maesta, A. Braga, Challenges of the treatment of patients with gestational trophoblastic disease, Rev. Bras. Ginecol. Obstet. 34 (4) (2012) 143-146.

[8] A. Braga, M. Burlá, F. Freitas, E. Uberti, M. Viggiano, S.Y. Sun, et al., Centralized coordination of decentralized assistance for patients with gestational trophoblastic disease in Brazil. A viable strategy for developing countries, J. Reprod. Med. 61 (5-6) (2016) 224-229.

[9] Fédération Internationale de Gynécologie et d'Obstétrique Oncology Committee, FIGO staging for gestational trophoblastic neoplasia 2000, Int. J. Gynaecol. Obstet. 77 (3) (2002) 285-287.

[10] R. Agarwal, S. Teoh, D. Short, R. Harvey, P.M. Savage, M.J. Seckl, Chemotherapy and human chorionic gonadotropin concentrations 6 months after uterine evacuation of molar pregnancy: a retrospective cohort study, Lancet 379 (9811) (2012) 130-135.

[11] A.M. Gillespie, S. Kumar, B.W. Hancock, Treatment of persistent trophoblastic disease later than 6 months after diagnosis of molar pregnancy, Br. J. Cancer 82 (8) (2000) 1393-1395.
[12] S.A. Khanlian, H.O. Smith, L.A. Cole, Persistent low levels of human chorionic gonadotropin: a premalignant gestational trophoblastic disease, Am. J. Obstet. Gynecol. 188 (5) (2003) 1254-1259.

[13] L.A. Cole, S.A. Khanlian, A. Giddings, S.A. Butler, C.Y. Muller, C. Hammond, et al., Gestational trophoblastic disease: 4 . Presentation with persistent low positive human chorionic gonadotropin test results, Gynecol. Oncol. 102 (2) (2006) 165-172.

[14] X.Q. Qian, L.L. Chen, B.H. Li, X.D. Cheng, X.Y. Wan, Long-term outcome of patients with persistent low-level elevation of human chorionic gonadotropin, J. Obstet. Gynaecol. Res. (2016), http://dx.doi.org/10.1111/jog.12953.

[15] F. Taylor, D. Short, R. Harvey, M.C. Winter, J. Tidy, B.W. Hancock, et al., Late spontaneous resolution of persistent molar pregnancy, BJOG (2016), http://dx.doi.org/10. 1111/1471-0528.13867.

[16] P.A. Bolze, J. Attia, J. Massardier, M.J. Seckl, L. Massuger, van Trommel, et al., Formalised consensus of the European Organisation for Treatment of Trophoblastic Diseases on management of gestational trophoblastic diseases, Eur. J. Cancer 51 (13) (2015) 1725-1731.

[17] H.Y.S. Ngan, E.I. Kohorn, L.A. Cole, R.J. Kurman, S.J. Kim, J.R. Lurain, et al., FIGO Cancer Report 2012. Trophoblastic disease, Int. J. Gynecol. Obstet. 119 (Suppl. 2) (2012) S130-S136.

[18] H.Y.S. Ngan, M.J. Seckl, R.S. Berkowitz, Y. Xiang, F. Golfier, P.K. Sekharan, et al., Update on the diagnosis and management of gestational trophoblastic disease, Int. J. Gynecol. Obstet. 131 (Suppl. 2) (2015) S123-S126.

[19] K.D. Bagshawe, Risk and prognostic factors in trophoblastic neoplasia, Cancer 38 (3) (1976) 1373-1385.

[20] T. Powles, A. Young, A. Sanitt, J. Stebbing, D. Short, M. Bower, et al., The significance of the time interval between antecedent pregnancy and diagnosis of high-risk gestational trophoblastic tumours, Br. J. Cancer 95 (9) (2006) 1145-1147.

[21] P. Schmid, Y. Nagai, R. Agarwal, N. Hancock, P.M. Savage, N.J. Sebire, et al., Prognostic markers and long-term outcome of placental-site trophoblastic tumours: a retrospective observational study, Lancet 374 (9683) (2009) 48-55.

[22] S.J. Kim, S.N. Bae, J.H. Kim, C.T. Kim, K.T. Han, J.M. Lee, et al., Effects of multiagent chemotherapy and independent risk factors in the treatment of high-risk GTT 25 years experiences of KRI-TRD, Int. J. Gynaecol. Obstet. 60 (Suppl. 1) (1998) S85-S96. 